May 2016

\title{
Assessment of WT1 expression as a marker of treatment outcome in karyotype normal acute myeloid leukemia patients in Pakistan
}

Zeeshan Ansar Ahmed

Aga Khan University

Muhammad Shariq Shaikh

Tariq Moatter

Follow this and additional works at: http://ecommons.aku.edu/

pakistan_fhs_mc_pathol_microbiol

Part of the Pathology Commons

\section{Recommended Citation}

Ahmed, Z., Shaikh, M., Moatter, T. (2016). Assessment of WT1 expression as a marker of treatment outcome in karyotype normal acute myeloid leukemia patients in Pakistan. JCPSP: Journal of the College of Physicians and Surgeons Pakistan, 26(5), 441-442.

Available at: http://ecommons.aku.edu/pakistan_fhs_mc_pathol_microbiol/459 


\title{
Assessment of WT1 Expression as a Marker of Treatment Outcome in Karyotype Normal Acute Myeloid Leukemia Patients in Pakistan
}

\author{
Zeeshan Ansar Ahmed, Muhammad Shariq Shaikh and Tariq Moatter
}

\begin{abstract}
Currently, there is an effort to predict relapse by follow-up monitoring of MRD and subsequently to begin the treatment of the patients during their clinical and hematological remission prior to overt hematological relapse. Expression of WT1 in AML is known to be independently associated with significant inferior response to therapy and short survival outcome. Follow-up monitoring of WT1 gene expression during or after therapy would be a valuable predictive marker for early recurrence or relapse of AML disease. This pilot study evaluated newly diagnosed and post-induction or consolidation chemotherapy of AML patients who were registered with the Oncology Clinics of the Aga Khan University Hospital, Karachi. High WT1 burden (> 5000 copies $/ \mathrm{ml}$ ) in 2 patients was indicative of early recurrence of the disease along with shorter disease-free and overall survival. Low WT1 expression $(<200$ copies $/ \mathrm{ml})$ in 2 patients after induction and consolidation therapy, respectively, was suggestive of better prognosis.
\end{abstract}

Key Words: Acute myeloid leukemia. Minimal residual disease. Wilm's tumor gene 1 (WT1). Prognosis.

The diagnosis and treatment of acute myeloid leukemia $(\mathrm{AML})$ has been a challenge for clinicians because of multiple pathogenic origins, especially genetic mutations which can influence its diagnosis, prognosis and treatment outcomes. In the last $10-15$ years, several novel genetic markers were introduced for AML to support its diagnosis, prognostication and monitoring of treatment efficacy. These makers are categorized into favourable and unfavourable, based on disease outcome, and are used for predicting disease progression at the time of diagnosis as well as response to chemotherapy. ${ }^{1}$

In current practice, molecular detection of MRD by PCR is the most specific and sensitive method. Molecular identification of MRD has increased the disease progression-free survival and overall survival of $A M L$ patients by predicting the relapse of disease, thus providing opportunity to the clinician to re-examine patients during their clinical and hematological remission period prior to overt hematological relapse. Treatment of MRD has a greater probability of success, is less distressing for the patients, and also has a lower cost than the treatment of an overt hematological relapse. ${ }^{2}$

Wilm's tumor (WT1) gene expression appears to be a highly promising molecular marker for MRD in leukemic patients, particularly in AML. WT1 gene is located on chromosome $11 \mathrm{p} 13$ and encodes a zinc finger transcription factor. It is implicated in cell proliferation

Department of Pathology and Laboratory Medicine,

The Aga Khan University Hospital, Karachi.

Correspondence: Dr. Zeeshan Ansar Ahmed, Assistant Professor, Department of Pathology and Laboratory Medicine,

The Aga Khan University Hospital, Stadium Road, Karachi. E-mail: zeeshanulqadr@gmail.com

Received: July 01, 2015; Accepted: December 10, 2015. and differentiation. WT1 is known to inhibit apoptosis via TP53 and BCL2. In many leukemic patients at the time of diagnosis, WT1 is highly expressed and it plays a significant role in leukomogenesis. ${ }^{3}$

WT1 expression is markedly low in cells of normal healthy individuals, with the exception of the CD 34+ hematopoietic progenitors. The expression of WT1 gene in leukemic cell is much higher than in normal bone marrow or peripheral cells. Consequently, this gene is suitable as a marker for monitoring MRD in AML either in bone marrow or peripheral blood samples, particularly in patients with normal cytogenetics or without specific marker identified. 4 Several studies have documented promising role of WT1 as an indicator of treatment outcome in AML. ${ }^{5}$ However, literature review revealed scarcity of data, addressing the role of this important marker in Pakistani AML patients.

In a pilot study, the authors quantified WT1 levels by RT $\mathrm{PCR}$ in 5 male patients with AML. All patients had normal karyotype and their mean age was 32 years (16 - 45). According to FAB classification, 2 patients were diagnosed as AML-M1, whereas 3 had AML-M2. All patients were in different phases of treatment at the time of analysis; 2 treatment naïve, 2 completed their induction phase, and 1 had completed consolidation phase. For WT1 expression analysis, $5 \mathrm{~mL}$ of whole blood was collected in an EDTA containing tube from each participant. The samples were transferred immediately to the laboratory for processing and preservation of nucleic acid (RNA) at $-8^{\circ} \mathrm{C}$.

For WT1 analysis, complementary DNA (cDNA) was synthesized from RNA extracted from patient samples. This cDNA was utilized for amplification by real time PCR using primers specific for WT1 gene. Standards and controls were run in parallel for ensuring quality 
assurance. WT1 gene expression was quantified using a validated standard curve, normalized with $A B L$ (internal control) copy number and ratio of WT1 copies/ABL copies. WT1 levels were expressed as copies $/ \mathrm{mL}$ of blood and also as ratio of WT1/ABL. The standard curves were generated by serial dilution of plasmid DNA containing insert of WT1 gene. For the assessment of quality parameters, positive and negative controls were included in every batch of assay.

Relatively higher level of WT1 mRNA (4000 - 9500 copies $/ \mathrm{ml}$ ) was detected in treatment naïve patients, whereas only 100 - 250 copies $/ \mathrm{ml}$ were seen in 2 patients (one was receiving induction and the other patient was on consolidation therapy). In addition, one patient who received induction therapy showed raised WT1 mRNA levels (7700 copies $/ \mathrm{ml}$ ).

WTI expression correlates with disease status both at the time of diagnosis and then different phases of the treatment. More than 5000 copies of $\mathrm{WTI} / \mathrm{ml}$ were detected in one treatment naïve and in one patient after induction chemotherapy. High WT1 burden in both these patients is indicative of early recurrence of the disease along with shorter disease-free and overall survival. The low WT1 expression (<200 copies/ml) in 2 patients after induction and consolidation therapy respectively was suggestive of better prognosis.

The significant copy number determent is yet to be established, however, a copy number of less than 200 is classified as lower level detection of MRD. The significance of WT1 in disease monitoring is well proven. However, absolute significant values are yet to be established as larger studies are required to establish accurate cut-off values to label MRD. Nevertheless, correlation of baseline values with follow-up levels provides significant insight in prognosis of the condition. ${ }^{6}$

This is confirmed in various studies that WTI is a good prognostic indicator in $80 \%$ of $A M L$ for disease monitoring and represented as name of "panleukemic marker". Since WT1 expression reflects the burden of more immature leukemic cells, it offers the advantage of easier and more stringent evaluation over time. 6 In WHO 2008 AML classification FLT3-ITD, CEBPA, NPM1,
RUNX1-RUNXT1 (translocation 8; 21) and core binding factor with (inversion and translocation of 16;16) are already established for prognostication. However, all these markers are tested only at the time of initial diagnosis. WT1 has an additional advantage of being utilized as an indicator of MRD. Therefore, baseline assessment along with follow-up levels provides better assessment of the disease behavior.

To date, majority of the studies published for the role of WT1 as an indicator of treatment outcome was conducted in Western countries; whereas, few studies from India, Iran and Middle Eastern countries are also available. To the best of the authors' knowledge, current study is the first one to be reported from Pakistan. Since, the sample size is small, biological difference of the disease in Pakistani population cannot be commented. Larger studies are required in this regard to address the variation in Pakistani population.

\section{REFERENCES}

1. Vardiman JW, Thiele J, Arber DA, Brunning. The 2008 revision of the World Health Organization (WHO) classification of myeloid neoplasms and acute leukemia: rationale and important changes. Blood 2009; 114:937-51.

2. Miglino M, Colombo N, Pica G, Grasso R, Clavio M. WT1 over expression at diagnosis may predict favorable outcome in patients with de novo non-M3 acute myeloid leukemia. Leukem Lymph 2011; 52:1961-9.

3. Qin Y, Zhu H, Jiang B, Li J, Lu X. Expression patterns of WT1 and PRAME in acute myeloid leukemia patients and their usefulness for monitoring minimal residual disease. Leuk Res 2009; 33:384-90.

4. Sakamoto $Y$, Mariya $Y$, Sasaki S. WT1 mRNA level in peripheral blood is a sensitive biomarker for monitoring minimal residual disease in acute myeloid leukemia. Tohoku $\mathrm{J}$ Exp Med 2009; 219:169-76.

5. Aref S, El-Sharawy, Sabry M, Raouf DA. Prognostic relevance of Wilms tumor 1 (WT1) gene Exon 7 mutations in-patient with cytogenetically normal acute myeloid leukemia. Indian $J$ Hematol Blood Transfuse 2013; 10:1007-13.

6. Pol'ak J, H'ajkov'a H, Maalaufov'a-Soukupov'a J. Estimation of molecular upper remission limit for monitoring minimal residual disease in peripheral blood of acute myeloid leukemia patients by WT1 expression. Exp Ther Med 2012; 3:129-33. 